\title{
Alfabetización estadística y probabilística: primeros pasos para su desarrollo desde la Educación Infantil
}

Claudia Vásquez Ortiz* Danilo Díaz-Levicoy**

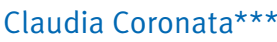
Ángel Alsina****

* (Pontificia Universidad Católica de Chile - PUC, Villarrica, Chile)

** (Universidad de Granada - UGR, Granada, España)

*** (Pontificia Universidad Católica de Chile - PUC, Villarrica, Chile)

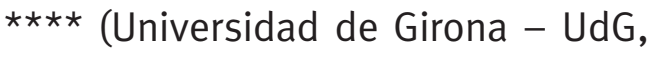
Girona, España)
Resumen: En este trabajo indagamos sobre las ideas fundamentales vinculadas a alfabetización estadística y probabilística que se pueden desarrollar desde la Educación Infantil. Para ello, analizamos las expectativas de aprendizaje explícitas en las directrices curriculares americanas y chilenas para dicho nivel educativo, las cuales se contrastan con los componentes cognitivos y de disposición presentes en el modelo de alfabetización estadística y probabilística. Asimismo, se ejemplifican diferentes situaciones para el desarrollo de la alfabetización estadística y probabilística en las primeras edades, como una forma de iniciar a los niños en el pensamiento crítico. Finalizamos con algunas orientaciones que pueden servir de apoyo a la formación del profesorado.

Palabras clave: Educación estadística. Formación del profesorado. Enseñanza preescolar. 


\section{INTRODUCCIÓN}

En las últimas décadas diversos autores han aportado recomendaciones para que el profesorado pueda llevar a cabo una enseñanza eficaz de la estadística y la probabilidad en el aula (e.g. GODINO; BATANERO; CAÑIZARES, 1987; NCTM, 2003; BATANERO; GODINO, 2004). En este artículo se asume que una enseñanza eficaz es aquella que requiere conocer lo que los estudiantes saben y lo que necesitan aprender, para luego estimularlos y ayudarlos para que lo aprendan bien (NCTM, 2003). Esto implica, por parte del profesorado: a) conocer y comprender en profundidad los conocimientos matemáticos que enseñan; b) conocer y comprender en profundidad a los estudiantes y, en especial, sus necesidades y posibilidades de aprendizaje; c) conocer y comprender en profundidad los recursos y estrategias docentes más adecuadas para llevar a cabo la enseñanza; y d) conocer y comprender en profundidad las formas de evaluar los conocimientos más acordes con los recursos y estrategias docentes usadas para llevar a cabo la enseñanza. En resumen, para una enseñanza eficaz es preciso que el profesorado disponga de un amplio abanico de conocimientos (disciplinares y didácticos), que permitan alfabetizar a los estudiantes, en el sentido que puedan usar de forma comprensiva y eficaz los conocimientos que aprenden en la escuela en todas las situaciones de la vida cotidiana en las que dichos conocimientos son necesarios (ALSINA, 2016). Para ello, el profesorado debería tener presentes las tres recomendaciones siguientes:

Las actividades informales de comparar, clasificar y contar pueden proporcionar a los niños los comienzos matemáticos para desarrollar la comprensión de los datos, del Análisis de datos y de la Estadística (...).

(...) los alumnos deberían ser capaces de organizar y mostrar sus datos a través de representaciones gráficas y resúmenes numéricos. Deberían realizar recuentos, registrándolos mediante palotes, tablas, diagramas de barras y diagramas de puntos. Los títulos y etiquetas utilizados en sus representaciones deberían identificar de forma clara qué datos se representan (...).

Las ideas sobre la probabilidad en estos niveles deberían ser informales, y centrarse en los juicios que emiten los alumnos con base en sus propias experiencias (NCTM, 2003, p. 113).

Desde esta perspectiva, la enseñanza de la estadística y la probabilidad requiere considerar, desde las primeras edades, el reconocimiento, la organización, la representación y la interpretación de datos. Se destaca que es importante, desde el principio, etiquetar adecuadamente tanto la variable de estudio como los distintos valores que toma la variable. Respecto 
a la probabilidad, se sugiere que el inicio de su enseñanza debería partir de situaciones de incertidumbre cercanas a la realidad de los estudiantes. A través de estas situaciones se debería empezar a introducir lenguaje específico para designar la posibilidad de ocurrencia de un determinado suceso (imposible, probable, seguro, etc.), considerando de esta forma el significado intuitivo de la probabilidad (ALSINA; VÁSQUEZ, 2016). Con ello se estará contribuyendo al desarrollo de ciudadanos alfabetizados estadística y probabilísticamente "capaces de hacer frente a una amplia gama de situaciones del mundo real que implican la interpretación o la generación de mensajes probabilísticos, así como la toma de decisiones” (GAL, 2005, p. 40). Para conseguir este propósito, es fundamental el desarrollo de nociones básicas de estadística y probabilidad, de manera informal desde las primeras edades. Este planteamiento coincide plenamente con las recomendaciones centrales del documento Guidelines for Assessment and Instruction in Statistics Education (GAISE, 2016): a) enseñar pensamiento estadístico; b) centrarse en la comprensión conceptual; c) integrar datos reales con un contexto y un propósito; d) fomentar el aprendizaje activo; e) usar la tecnología para explorar conceptos y analizar datos; y f) usar evaluaciones para mejorar y evaluar el aprendizaje de los estudiantes. Estas recomendaciones han sido recientemente reordenadas, revisadas y ampliadas sugiriendo dos nuevos énfasis específicos - que reflejan la práctica moderna y aprovechamiento de las tecnologías disponible - para lo refiere a la enseñanza del pensamiento estadístico (GAISE, 2016, p. 6):

La enseñanza como un proceso de investigación para la resolución de problemas y la toma de decisiones. Los estudiantes no deben percibir erróneamente la estadística como una colección no relacionada de fórmulas y métodos. Por el contrario, los estudiantes deben comprender que las estadísticas son un proceso de resolución de problemas y toma de decisiones que es fundamental para la investigación científica y esencial para tomar decisiones acertadas.

La enseñanza de brindar a los estudiantes experiencias vinculadas con el pensamiento multivariable, puesto que vivimos en un mundo complejo en el que la respuesta a una pregunta a menudo depende de muchos factores. Los estudiantes encontrarán tales situaciones dentro de sus propios campos de estudio y de la vida cotidiana. Hay que preparar a los estudiantes para responder preguntas desafiantes que les exijan que investiguen y exploren las relaciones entre muchas variables. Hacerlo les ayudará a apreciar el valor del pensamiento y los métodos estadísticos.

En definitiva, se trata de ofrecer a los estudiantes herramientas para contestar a preguntas cuyas respuestas no son inmediatas, a la vez que les faciliten la toma de decisiones en situaciones de incertidumbre. Con este propósito 
en mente el NCTM (2003) propone que a partir de los 3 años y durante toda la etapa escolar el proceso de enseñanza y aprendizaje de la estadística y probabilidad se realice de manera gradual y continua, en torno a:

- formular preguntas que pueden ser dirigidas con datos y recolectar, organizar y desplegar datos relevantes para responderlas;

- seleccionar y usar métodos estadísticos apropiados para analizar datos;

- desarrollar y evaluar inferencias y predicciones que estén basadas en datos; $y$

- entender y aplicar conceptos básicos de probabilidad.

Desde este prisma, en este trabajo se realiza, en primer lugar, una revisión de las orientaciones curriculares nacionales e internacionales para la enseñanza de la estadística y la probabilidad en las primeras edades, a la luz del modelo de alfabetización estadística y probabilística propuestos por Gal $(2002,2005)$. En segundo lugar se presentan diversas experiencias de aula para llevar a cabo una enseñanza eficaz de la estadística y la probabilidad en Educación Infantil "que involucre a los estudiantes en un aprendizaje significativo mediante experiencias individuales y colaborativas que fomenten su habilidad para dar sentido a las ideas matemáticas y para razonar de una manera matemática" (NCTM, 2015, p. 7). A través de estas experiencias se pretende que los estudiantes de Educación Infantil comiencen a aprender desde pequeños conocimientos que les sirvan de base para que progresivamente sean ciudadanos bien informados y consumidores inteligentes. Finalmente, se exponen algunas implicaciones y orientaciones que pueden servir de apoyo al profesorado de Educación Infantil para fomentar la alfabetización estadística y probabilística de los estudiantes desde las primeras edades de escolarización.

\section{Alfabetización estadística y pRobabilística}

De acuerdo con el planteamiento de Gal (2002), la alfabetización estadística se refiere a la capacidad para interpretar, evaluar críticamente y, cuando sea pertinente, expresar opiniones respecto a la información estadística, los argumentos relacionados con los datos o fenómenos estocásticos. Para ello, se requiere de la activación conjunta de componentes cognitivos y de disposición, como se observa en el Cuadro 1. 
Cuadro 1 - Elementos cognitivos y de disposición para el desarrollo de la alfabetización estadística

\begin{tabular}{|c|c|}
\hline Elementos cognitivos & Elementos disposicionales \\
\hline $\begin{array}{l}\text { Habilidades de alfabetización } \\
\text { - Conocimientosestadísticos (incluyendo } \\
\text { también algunos conocimientos } \\
\text { probabilísticos, incluso informal). } \\
\text { - Conocimiento matemático contextual o } \\
\text { del mundo del conocimiento. } \\
\text { - Plantearse el conocimiento de } \\
\text { cuestiones críticas }\end{array}$ & $\begin{array}{l}\text { Presencia de una posición crítica, es decir, la voluntad } \\
\text { de adoptar actitudes cuestionando ciertas creencias: } \\
\text { - La creencia en el poder de los procesos } \\
\text { estadísticos. } \\
\text { - La creencia en sí mismo como persona capaz de } \\
\text { pensar estadísticamente. } \\
\text { - La creencia en la legitimidad de la adopción } \\
\text { de una perspectiva crítica sobre información } \\
\text { recibida de fuentes "oficiales" o de expertos. }\end{array}$ \\
\hline
\end{tabular}

Fuente: Gal (2002).

Por su parte, Gal (2005) caracteriza la alfabetización probabilística, vinculándola a la alfabetización estadística, como la capacidad de acceder, utilizar, interpretar y comunicar información e ideas relacionadas con la probabilidad, con el fin de participar y gestionar eficazmente las demandas de las funciones y tareas que implican incertidumbre y riesgo del mundo real. Lo que a su vez también requiere de la activación conjunta de componentes cognitivos y de disposición (Cuadro 2).

Cuadro 2 - Elementos cognitivos y de disposición para el desarrollo de la alfabetización probabilística

Elementos cognitivos

- Las grandes ideas: variación, aleatoriedad, independencia, previsibilidad/incertidumbre.

- Lenguaje: los términos y los métodos usados para comunicar acerca del grado de incertidumbre.

- Cómo calcular las probabilidades.

- La comprensión del papel y las implicaciones de los problemas probabilísticos y mensajes en diferentes contextos y en el discurso personal y público.

- Preguntas críticas: Cuestiones para reflexionar cuando se trata de probabilidades.w

\section{Elementos disposicionales}

- Postura crítica ante mensajes cuantitativos que pueden ser engañosos, unilaterales, sesgados o incompletos.

- Visión positiva de sí mismo como individuo capaz de realizar razonamientos probabilísticos en situaciones de incertidumbre que sean relevantes.

- Capacidad para evaluar el riesgo asociado con los eventos o resultados de relevancia para la vida. 
Aun cuando estos componentes son presentados por separado, todos ellos interactúan entre sí de manera compleja durante el aprendizaje real (GAL, 2005). Esto significa que una instrucción que se centre sólo en uno o dos elementos no será suficiente para desarrollar un "comportamiento alfabetizado de estadística y probabilidad", por lo que es esencial que "se vayan adquiriendo a lo largo de la escolarización, a través de una planificación que considere los conocimientos que se deberían enseñar y una gestión que tenga presente las formas más eficaces de enseñarlos” (ALSINA, 2017, p. 29). Pero, ¿Cómo desarrollar la alfabetización estadística y probabilística desde las primeras edades? ¿Qué aspectos se deben considerar? En relación con los contextos de enseñanza y aprendizaje, para poder plantear tareas auténticas que fomenten la alfabetización estadística y probabilística desde las primeras edades, un buen punto de partida son las orientaciones específicas propuestas por Alsina (2017, p. 30-31):

a) Contenidos de estadística y probabilidad de 3 a 4 años:

- Identificación de datos muy sencillos del entorno cercano a partir de variables discretas.

- Representación de los datos identificados, principalmente con dibujos.

- Identificación posterior de la frecuencia absoluta de cada valor a partir del recuento.

- Reconocimiento de la posibilidad de ocurrencia de hechos: hechos seguros.

- Organización de los datos identificados, principalmente a través de clasificaciones, para facilitar la comparación y la representación.

- Comparación e interpretación de los datos representados con dibujos, usando los comparativos "más ... que", "menos ... que", "tanto ... como".

- Resolución de situaciones aritméticas elementales a partir de los datos representados.

b) Contenidos de estadística y probabilidad de 4 a 5 años:

- Identificación de datos más complejos a partir de variables discretas.

- Representación de los datos identificados, principalmente con objetos.

- Identificación posterior de la frecuencia absoluta de cada valor a partir del recuento. Reconocimiento de la posibilidad de ocurrencia de hechos: hechos imposibles.

- Organización de los datos identificados, principalmente a través de clasificaciones, para facilitar la comparación y la representación.

- Comparación e interpretación de los datos representados con objetos, 
usando los comparativos "más ... que", "menos ... que", "tanto ... como"

- Comparación de la posibilidad de ocurrencia de hechos sencillos, según si son seguros o imposibles.

- Composición y descomposición de las frecuencias absolutas.

- Resolución de situaciones aritméticas algo más complejas a partir de los datos representados.

c) Contenidos de estadística y probabilidad de 5 a 6 años:

- Identificación de datos cada vez más complejos a partir de variables discretas. Representación de los datos identificados en gráficos de barras, con una correspondencia término a término.

- Identificación posterior de la frecuencia absoluta de cada valor a partir del recuento. Reconocimiento de la posibilidad de ocurrencia de hechos: hechos probables.

- Organización de los datos identificados, principalmente a través de clasificaciones, para facilitar la comparación y la representación.

- Comparación e interpretación de los datos representados en gráficos de barras. Comparación de la posibilidad de ocurrencia de hechos sencillos, según si son imposibles, probables o seguros.

- Composición y descomposición de las frecuencias absolutas.

- Resolución de situaciones aritméticas más complejas a partir de los datos representados en un gráfico de barras.

\section{3. ¿QUÉ DICEN LAS ORIENTACIONES CURRICULARES?}

Actualmente existe un acuerdo generalizado en que la estadística y la probabilidad, debido a sus múltiples aplicaciones en distintas áreas del saber, es parte importante de la matemática, por lo que es necesario que el pensamiento estadístico y probabilístico se desarrolle desde las primeras edades (nivel parvulario). Esta tendencia ha sido adoptada por el NCTM desde 1989, al incluir como área temática en el Curriculum and Evaluation Standard for School Mathematics a Datos y Azar (NCTM, 1989), iniciativa que desde entonces ha cobrado fuerza y se ha plasmado, últimamente, en los Principles and Standard for School Mathematics (NCTM, 2000). Por medio de tales Principios y Estándares, se busca “describir las características particulares de una educación matemática de gran calidad”, además de “describir los contenidos y procesos matemáticos que deberían aprender los estudiantes" (NCTM, 2003, p. 11). En lo que respecta al estándar de contenido de "Análisis de datos y Probabilidad" el NCTM (2003) propone abordar conceptos básicos 
y distintas aplicaciones de la estadística y las probabilidades que deben permitir establecer, de manera progresiva, conexiones entre la matemática y otros ámbitos del saber, así como con experiencias de la vida diaria. Desde esta perspectiva, propone iniciar el estudio de la estadística y la probabilidad a partir de la Educación Infantil, como un continuo, hasta el bachillerato, de manera tal que se favorezca la adquisición progresiva de los contenidos descritos en el Cuadro 3:

Cuadro 3 - Contenidos de estadística y probabilidad de Pre K-2 (3 a 8 años)

Contenidos de estadística de Pre K-2 (3 a 8 años)

\begin{tabular}{|c|c|c|c|}
\hline $\begin{array}{l}\text { Formular preguntas que puedan } \\
\text { abordarse con datos y recoger, } \\
\text { organizar y presentar datos } \\
\text { relevantes para responderlas }\end{array}$ & $\begin{array}{l}\text { Seleccionar y } \\
\text { utilizar métodos } \\
\text { estadísticos } \\
\text { apropiados para } \\
\text { analizar datos }\end{array}$ & $\begin{array}{l}\text { Desarrollar y } \\
\text { evaluar inferencias } \\
\text { y predicciones } \\
\text { basadas en datos }\end{array}$ & $\begin{array}{l}\text { Comprender y } \\
\text { aplicar conceptos } \\
\text { básicos de } \\
\text { probabilidad }\end{array}$ \\
\hline $\begin{array}{l}\text { Proponer preguntas y recoger } \\
\text { datos relativos a ellos y su } \\
\text { entorno. } \\
\text { Ordenar y clasificar datos de } \\
\text { acuerdo con sus atributos y } \\
\text { organizar datos relativos a } \\
\text { aquellos. } \\
\text { Representar datos mediante } \\
\text { objetos concretos, dibujos y } \\
\text { gráficos. }\end{array}$ & $\begin{array}{l}\text { Describir parte } \\
\text { de los datos y el } \\
\text { conjunto total de } \\
\text { los mismos para } \\
\text { determinar lo } \\
\text { que muestran los } \\
\text { datos. }\end{array}$ & $\begin{array}{l}\text { Discutir sucesos } \\
\text { probables e } \\
\text { improbables } \\
\text { relacionados con } \\
\text { las experiencias de } \\
\text { los alumnos. }\end{array}$ & \\
\hline
\end{tabular}

Fuente: NCTM (2003).

Como se observa, el desarrollo de los conceptos básicos de estadística y probabilidad se inicia de manera informal, gradual y progresiva en los primeros niveles, con un fuerte desarrollo de los conceptos y procedimientos involucrados, de modo que al finalizar la etapa escolar los estudiantes cuenten con un sólido conocimiento sobre estos temas. En definitiva, se trata de entregar a los estudiantes las herramientas necesarias para desarrollar y alcanzar una alfabetización estadística y probabilística, que facilite el desarrollo de un pensamiento crítico, que les permita comprender y comunicar distintos tipos de información en numerosas situaciones de la vida diaria en que los fenómenos aleatorios, el azar y la incertidumbre están presentes.

Chile no es ajeno a la necesidad de desarrollar, desde las primeras edades, ciudadanos críticos, alfabetizados estadística y probabilísticamente, por lo que en la Ley General de Educación para la Educación Primaria, se refiere 
explícitamente a la necesidad de que:

(...) los educandos desarrollen los conocimientos, habilidades y actitudes que les permitan: pensar en forma reflexiva, evaluando y utilizando información y conocimientos, de manera sistemática y metódica, para la formulación de proyectos y resolución de problemas; comprender y utilizar conceptos y procedimientos matemáticos básicos en la resolución de problemas cotidianos, y apreciar el aporte de la matemática para entender y actuar en el mundo" (MINEDUC, 2009, p. 10).

En respuesta a dicho planteamiento, en las actuales Bases Curriculares de la Educación Parvularia (MINEDUC, 2018), si bien no se señalan de manera explícita contenidos vinculados al estudio de la estadística y probabilidad, se observan en el núcleo de pensamiento matemático (cuyo propósito es potenciar en los niños las habilidades, actitudes y conocimientos relacionados con el pensamiento lógico y los números, que les posibiliten comunicar y resolver situaciones prácticas cotidianas) diversos objetivos de aprendizaje que de una u otra manera se relacionan con nociones básicas de la estadística y la probabilidad (ver Cuadro 4). 
Cuadro 4 - Contenidos relacionados con estadística y probabilidad

\begin{tabular}{|c|c|c|}
\hline $\begin{array}{l}\text { Primer Nivel } \\
\text { (Sala Cuna } \\
\text { de } 0 \text { a } 2 \\
\text { años) }\end{array}$ & $\begin{array}{l}\text { Segundo Nivel } \\
\text { (Medio de } 2 \text { a } 4 \text { años) }\end{array}$ & $\begin{array}{c}\text { Tercer Nivel } \\
\text { (Transición de } 4 \text { a } 6 \text { años) }\end{array}$ \\
\hline & $\begin{array}{l}\text { Emplear cuantificadores, tales } \\
\text { como: más/menos, mucho/ } \\
\text { poco, todo/ninguno, al comparar } \\
\text { cantidades de objetos en } \\
\text { situaciones cotidianas. } \\
\text { Emplear progresivamente } \\
\text { los números, para contar, } \\
\text { identificar, cuantificar y } \\
\text { comparar cantidades, hasta el } \\
10 \text { e indicar orden o posición } \\
\text { de algunos elementos en } \\
\text { situaciones cotidianas o juegos. } \\
\text { Representar progresivamente, } \\
\text { números y cantidades en forma } \\
\text { concreta y pictórica hasta el } 10 . \\
\text { Resolver progresivamente } \\
\text { problemas simples, de manera } \\
\text { concreta y pictórica, agregando o } \\
\text { quitando hasta } 5 \text { elementos. } \\
\text { Identificar algunas acciones que } \\
\text { se llevaron a cabo para resolver } \\
\text { problemas. }\end{array}$ & $\begin{array}{l}\text { Emplear cuantificadores, tales como: “más que”, } \\
\text { “menos que”, “igual que”, al comparar cantidades de } \\
\text { objetos en situaciones cotidianas. } \\
\text { Orientarse temporalmente en situaciones cotidianas, } \\
\text { empleando nociones y relaciones de secuencia } \\
\text { (antes/ahora/después/al mismo tiempo, día/noche), } \\
\text { frecuencia (siempre/a veces/nunca) y duración (larga/ } \\
\text { corta). } \\
\text { Emplear los números, para contar, identificar, } \\
\text { cuantificar y comparar cantidades hasta el } 20 \text { e } \\
\text { indicar orden o posición de algunos elementos en } \\
\text { situaciones cotidianas o juegos. } \\
\text { Representar números y cantidades hasta el 10, en } \\
\text { forma concreta, pictórica y simbólica. } \\
\text { Resolver problemas simples de manera concreta y } \\
\text { pictórica agregando o quitando hasta } 10 \text { elementos, } \\
\text { comunicando las acciones llevadas a cabo. } \\
\text { Emplear medidas no estandarizadas, para determinar } \\
\text { longitud de objetos, registrando datos, en diversas } \\
\text { situaciones lúdicas o actividades cotidianas. } \\
\text { Comunicar el proceso desarrollado en la resolución } \\
\text { de problemas concretos, identificando la pregunta, } \\
\text { acciones y posibles respuestas }\end{array}$ \\
\hline
\end{tabular}

Fuente: MINEDUC (2018).

A partir de las orientaciones curriculares nacionales e internacionales se observa que los gráficos estadísticos son un elemento clave para el desarrollo de la alfabetización estadística a partir de las primeras edades (ARTEAGA; BATANERO; CAÑADAS; CONTRERAS, 2011; BATANERO; ARTEAGA; RUIZ, 2010), puesto que su trabajo en el aula facilita la conexión entre disciplinas y contextos de vida cotidiana. De igual manera, el trabajo con estas representaciones en las primeras edades permiten potenciar el concepto de número, conteo, operaciones, etc., contribuyendo al desarrollo de otras competencias matemáticas vinculadas con el sentido numérico (ARTEAGA; BATANERO; CONTRERAS, 2011). En el caso de la alfabetización probabilística, el aprendizaje en las primeras edades se inicia de manera informal introduciendo vocabulario vinculado a las nociones de probabilidad por medio de actividades o situaciones problemas centradas en sus propias experiencias (GODINO; BATANERO; CAÑIZARES, 1987), llevándoles a 
responder preguntas sobre la posibilidad de ocurrencia de sucesos y que consideren el empleo de términos tales como: seguro, probable o imposible, entre otros. Por tanto, el lenguaje asociado a lo cotidiano constituye un elemento clave para alcanzar una progresión hacia un y avanzar así en la construcción del conocimiento probabilístico (VÁSQUEZ; ALSINA, 2017). En consecuencia, durante las primeras edades es fundamental proporcionar actividades que brinden oportunidades a los estudiantes de desarrollar un pensamiento crítico (AIZIKOVITSCH-UDI; KUNTZE, 2014), por lo que es preciso motivar el aprendizaje de la estadística y la probabilidad a partir de problemas provenientes de contextos de vida cotidiana, incentivando una actitud crítica frente a afirmaciones erróneas y abusos que a menudo aparecen en los medios de comunicación (FRANKLIN et al., 2005).

Este planteamiento ha cobrado gran importancia durante los últimos años, por su repercusión en la alfabetización estadística y probabilística de todo ciudadano inmerso en la era de la información y el consiguiente desarrollo que esto trae a los países. En este sentido, la enseñanza de la estadística y la probabilidad se debería considerar como algo transversal. El desafío es grande, y un buen punto de partida para potenciar la alfabetización estadística y probabilística es valerse de la utilidad y aplicaciones en diversas disciplinas, como ciencias exactas, biología, medicina o sociología, por mencionar sólo algunos ejemplos. Si bien el enfoque más utilizado y promocionado para la enseñanza de la estadística y la probabilidad se fundamenta en la toma de decisiones y predicciones basadas en datos, es necesario vincular dichos datos con contextos que sean significativos a los estudiantes, de modo tal que éstos planteen preguntas, obtengan conclusiones y evalúen conjeturas e investigaciones. De esta forma, se enfatiza la comprensión conceptual, la exploración de datos y el uso de tecnología apropiada, por encima de los procedimientos matemáticos de datos aislados y sin significado (WATSON, 2014).

\section{EXPERIENCIAS PARA LA ENSEÑANZA DE LA ESTADÍSTICA Y LA PROBABILIDAD} EN LA EDUCACIÓN INFANTIL

Dado que "existe todavía poca tradición de trabajar de forma sistemática el bloque de contenidos de estadística y probabilidad en Educación Infantil" (ALSINA, 2012, p. 5), es que en este apartado se presentan algunas experiencias para la enseñanza de estos temas en las primeras edades, a través de las cuales se pretende contribuir a su enseñanza a partir de contextos significativos y ajustados a las necesidades de los estudiantes de este nivel 
escolar. Estas experiencias se fundamentan en la propuesta de Alsina (2017), cuyo foco está en la identificación y la organización de los datos (sobre todo a través de la clasificación, aunque también se pueden realizar ordenaciones); la representación a través de objetos, dibujos o gráficos; y su posterior interpretación. Sin dejar de lado el trabajo a partir de la propia experiencia, ya sea propuesto por el profesor o bien por los propios estudiantes.

En lo que respecta a la probabilidad, se deben considerar que los estudiantes empiecen a usar de forma comprensiva lenguaje probabilístico elemental: imposible, probable y seguro a partir de sucesos inciertos que forman parte del entorno de los estudiantes, considerando de esta forma el significado intuitivo de la probabilidad (VÁSQUEZ, 2018), como se ha indicado anteriormente.

\section{Experiencia 1: Trabajando con sílabas y frecuencias}

- Nivel: 5-6 años

- Contenidos implícitos: esta actividad integra contenidos vinculados al lenguaje (conteo de las sílabas de una palabra) y a la recogida, organización, representación e interpretación de datos.

- Materiales: láminas con nombres de animales divididos en sílabas con números del 1 al 4.

- Habilidadesy aprendizajes a lograr: Con esta experiencia de aprendizaje se busca que los niños comparen, clasifiquen, organicen, representen y profundicen en la construcción de número en base a la información disponible. Pudiendo resolver problemas simples de manera concreta y pictórica al comunicar sus acciones llevadas a cabo.

- Gestión de la actividad:

En este caso, el profesor presenta distintas láminas con nombres de animales y va nombrando de acuerdo con sus sílabas y realizando el conteo del número de sílabas que tiene el nombre de cada animal. Posteriormente, solicita a los estudiantes que clasifiquen las láminas de acuerdo a la cantidad de sílabas que contiene cada nombre, por ejemplo a través de Diagramas de Venn, y que después ordenen los diagramas según la cantidad de nombres que contienen (Figura 1). Es importante que el profesor realice preguntas del tipo: ¿cuántos animales tienen 3 sílabas?; ¿hay más animales con cuatro o con tres sílabas?; ¿de qué otra manera se podrían representar los datos? Por medio de este tipo de preguntas se espera que los niños lleguen a representar la información contenida en los diagramas de Venn por medio de gráficos de barras simples. Finalmente, se les puede plantear la pregunta ¿qué se observa en el gráfico? para fomentar la interpretación de los datos representados. 
Figura 1: Trabajando con sílabas y frecuencias (archivo de los autores).
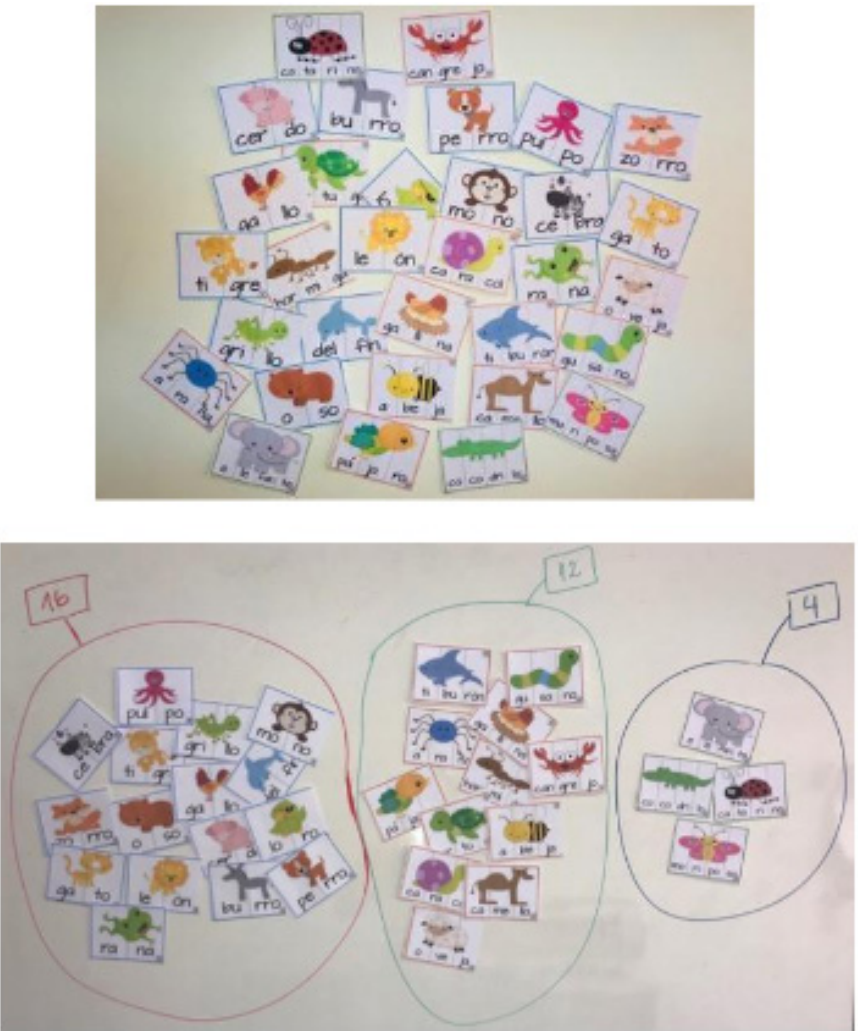

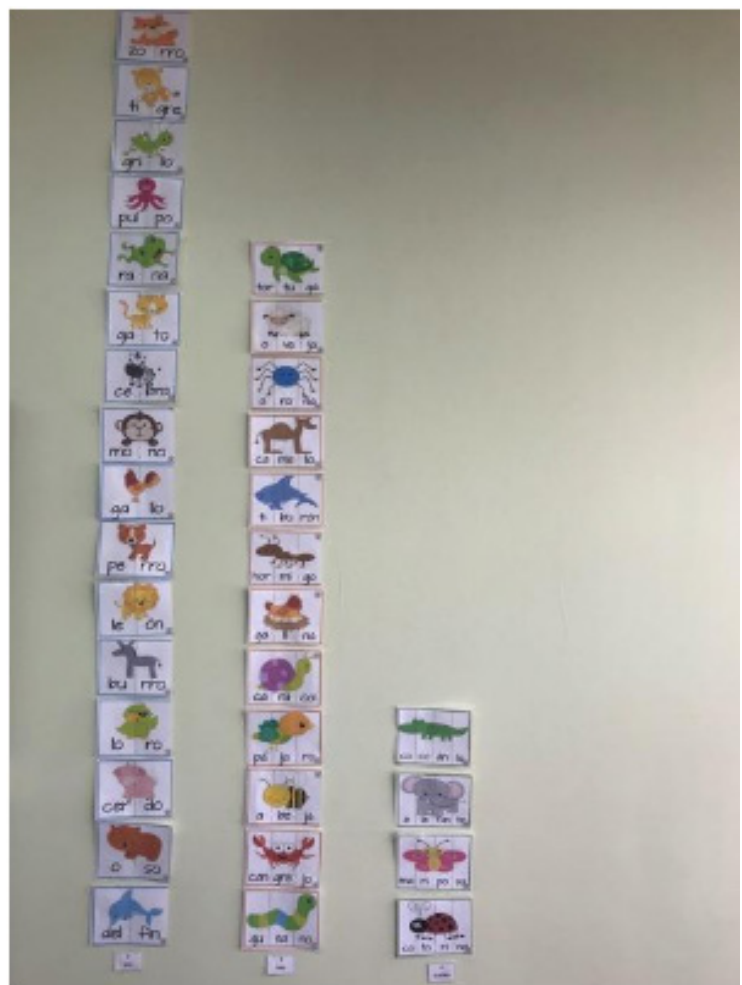

Experiencia 2: ¿Soleado, parcial, nublado, lluvioso, tormenta, nieve?

- Nivel: 3-4 años

- Contenidos implícitos: recogida, organización, representación e interpretación de datos; uso de lenguaje probabilístico elemental (imposible, probable, seguro, etc.).

- Materiales: diversas láminas con dibujos relacionados con el pronóstico del tiempo (soleado, lluvioso, parcial, nublado, tormenta, nieve) y tablero con los días de la semana (de lunes a viernes) para ir registrando el tiempo atmosférico del momento día a día.

- Habilidades y aprendizajes a lograr: Con este tipo de experiencia de aprendizaje se pretende que los niños logren desarrollar nociones y relaciones de frecuencia con un nivel inicial de representación, para poder conocer y explicarse más profundamente los acontecimientos de su medio. En este sentido, se busca que avancen en el logro de la representación numérica y de cantidades, en forma concreta, pictórica y simbólica.

- Gestión de la actividad:

El objetivo de esta actividad es que los estudiantes realicen una investigación estadística a partir del tiempo atmosférico y que se aproximen a las primeras nociones de probabilidad, por medio de los grados de posibilidad de un 
determinado suceso, utilizando lenguaje probabilístico elemental (imposible, probable, seguro). Asimismo, con este tipo de actividad se pretende que los estudiantes observen cómo los datos se acumulan a lo largo del tiempo, lo que permite realizar ciertas predicciones en torno a su comportamiento.

Para ello, el profesor utiliza el tablero del pronóstico del tiempo (Figura 2) y realiza preguntas que lleven a los estudiantes a interpretar datos y realizar predicciones acerca de estos, como por ejemplo: ¿cómo ha estado el tiempo atmosférico durante el mes de marzo?; ¿ha habido más días de sol o de lluvia?; ¿cuántos días ha llovido?; ¿es seguro, probable o posible que mañana sea un día soleado?; ¿por qué crees que sucederá eso?

Es importante que al final de cada semana (así como al final del mes) los niños contabilicen los registros realizados durante cada semana y registren la información en un gráfico.

Figura 2: Predicción del tiempo: ¿Soleado, parcial, nublado, lluvioso, tormenta, nieve? (archivo de los autores)
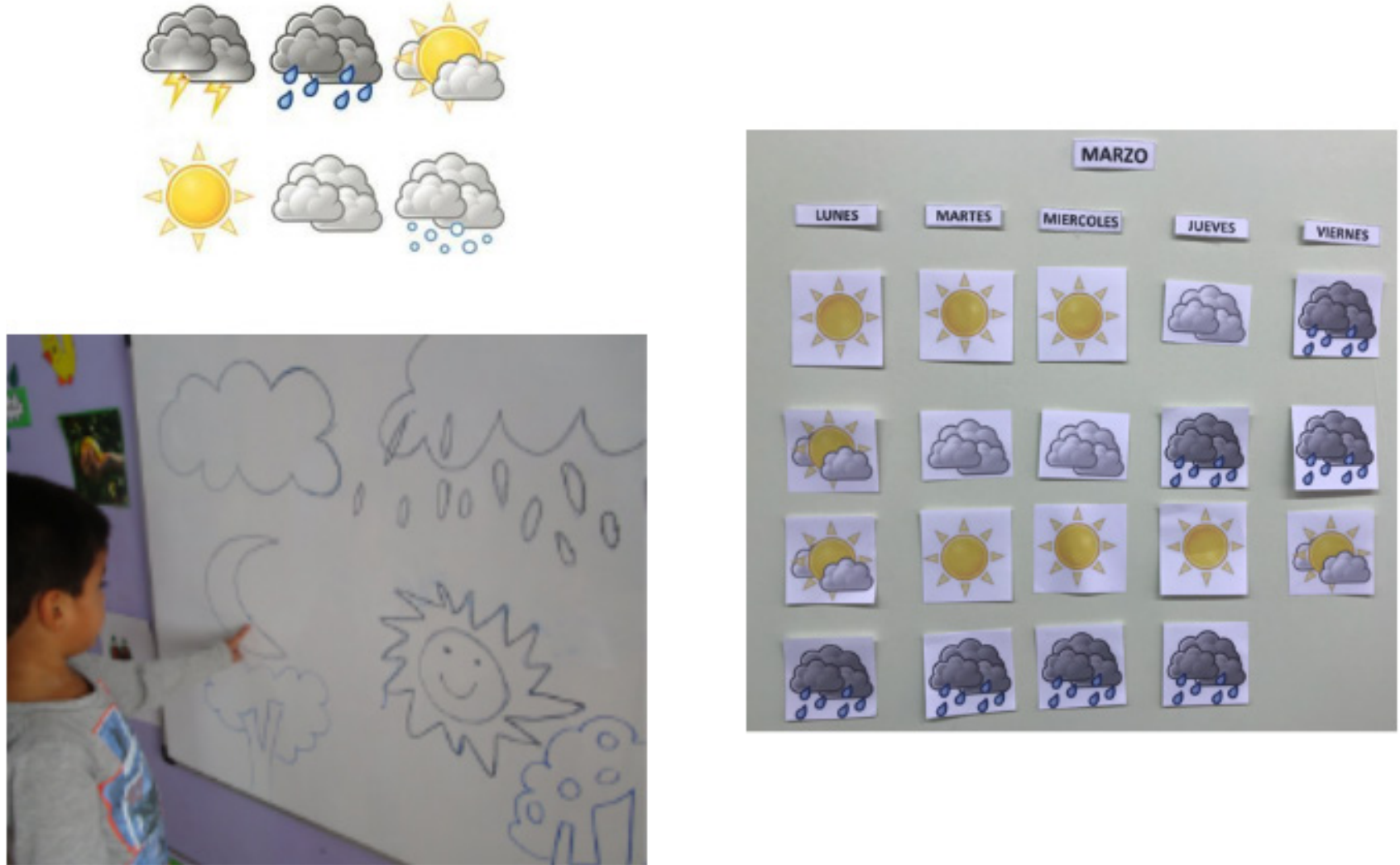
Experiencia 3: ¿Cuándo es tu cumpleaños?

- Nivel: 5-6 años

- Contenidos implícitos: recogida, organización, representación e interpretación de datos.

- Materiales: lámina con meses del año y fotografía (o distintivo) de cada niño del curso.

- Habilidades y aprendizajes a lograr: En esta experiencia de aprendizaje se desarrolla la habilidad de comunicar el proceso desarrollado en la resolución de problemas concretos, identificando la pregunta, acciones y posibles respuestas. Además la de emplear cuantificadores, tales como: "más que”, "menos que”, “igual que”, al comparar cantidades de objetos en situaciones cotidianas. Finalmente, se potencia la representación de números y cantidades en forma pictórica y simbólica.

- Gestión de la actividad:

Por medio de esta actividad se pretende que los niños realicen una investigación estadística acerca del mes de cumpleaños de sus compañeros de curso. Con esta finalidad, el profesor les pregunta ¿en qué mes están de cumpleaños? A continuación entrega a cada niño su fotografía (o distintivo) y les pide que la peguen (bajo su supervisión) en el panel donde se encuentran los meses del año. Seguidamente plantea diversas preguntas del tipo: ¿en qué mes no está de cumpleaños ningún niño del curso?; ¿cuál es el mes en que hay más cumpleaños?; ¿cuántos niños están de cumpleaños en verano?; ¿cuántos están de cumpleaños en invierno?; ¿cuál es el mes en que hay menos cumpleaños? Asimismo, puede preguntarles ¿de qué otra manera se puede representar esta información?; ¿existe otra forma de registrar estos datos? Por medio de estas preguntas se pretende que los niños identifiquen otras posibles formas de representar los datos, por ejemplo por medio de un diagrama de puntos como el que se muestra en la Figura 3.

No hay que olvidar que el rol del profesor es realizar buenas preguntas que comprometan a los estudiantes a que compartan deliberadamente ideas, enfoques y razonamiento matemáticos, utilizando diversas representaciones. 
Figura 3. Representación del mes de cumpleaños (archivo de los autores).

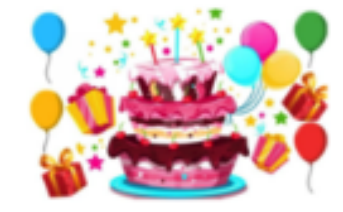

¿Cuándo es tu cumpleaños?

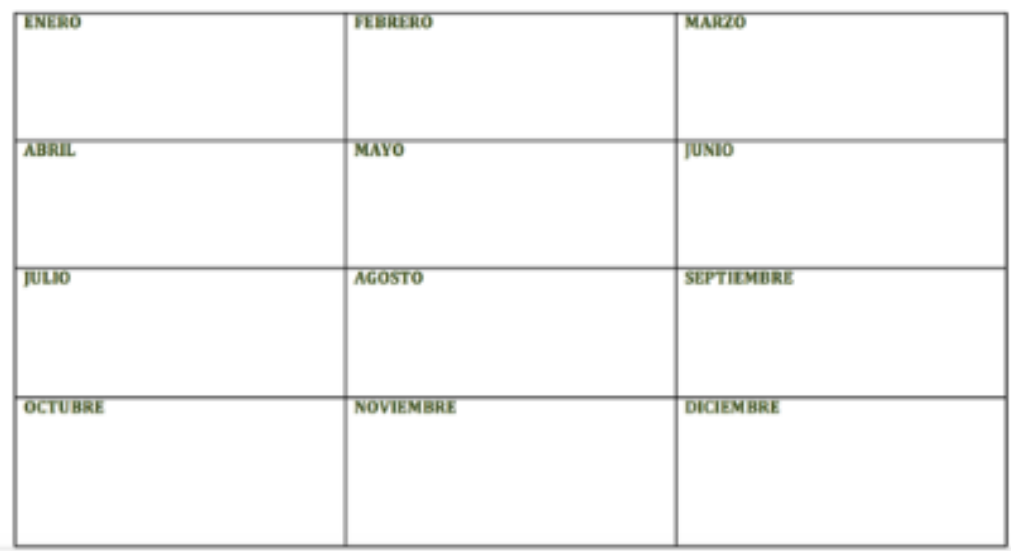

\begin{tabular}{|c|c|c|c|c|c|c|c|c|c|c|c|}
\hline & & & & & & & & & & $\bullet$ & \\
\hline & & & & & & & & & & $\bullet$ & \\
\hline & & & & & & & & & & $\bullet$ & \\
\hline$\bullet$ & & & & & & $\bullet$ & & & & $\bullet$ & \\
\hline$\bullet$ & $\bullet$ & & & $\bullet$ & & $\bullet$ & & $\bullet$ & & $\bullet$ & $\bullet$ \\
\hline$\bullet$ & $\bullet$ & $\bullet$ & & $\bullet$ & $\bullet$ & $\bullet$ & & $\bullet$ & $\bullet$ & $\bullet$ & $\bullet$ \\
\hline Enero & Fecbrero & Marzo & Mbrrl & Mayo & funlo & jullo & Agasto & Septiembre & Octubre & Noviembre & Dociembre \\
\hline
\end{tabular}

Experiencia 4:¿Cuántas letras tiene mi nombre?

- Nivel: 5-6 años

- Contenidos implícitos: recogida, organización, representación e interpretación de datos.

- Materiales: nombre de cada niño, ya sea escrito por ellos mismos o entregado por el profesor.

- Habilidades y aprendizajes a lograr: En esta experiencia se potencia en los niños el aprendizaje de resolver problemas simples de manera concreta y pictórica. Por otro lado, se trabaja la habilidad de comunicar el proceso desarrollado en la resolución de problemas concretos, 
identificando la pregunta, acciones y posibles respuestas. Finalmente, el emplear los números, para contar, identificar, cuantificar y comparar cantidades.

- Gestión de la actividad:

Por medio de esta actividad que conecta aspectos del lenguaje (reconocimiento de letras) con representación y análisis de datos, se pretende que los estudiantes recojan, organicen, representen y analicen datos provenientes de su curso. Para ello, el profesor les puede solicitar que escriban su nombre en un papel o bien entregarles un papel con su nombre ya escrito, y pedirles que contabilicen el número de letras que tiene su nombre. Seguidamente solicita que clasifiquen sus nombres según la cantidad de letras a través de un diagrama como el que se muestra en la Figura 4. Es importante que el profesor realice preguntas del tipo: ¿cuál es el nombre que tiene más/menos letras en nuestro grupo?; ¿cuántos nombres hay de cinco letras?; ¿hay más nombres con cuatro o con seis letras?; ¿cómo nos dimos cuenta de cuál es el nombre con más letras?; ¿qué hicimos para saber esto?; ¿cómo podemos organizar o representar los nombres?; etc.

Figura 4: Diagrama para representar la cantidad de letras de cada nombre (archivo de los autores)

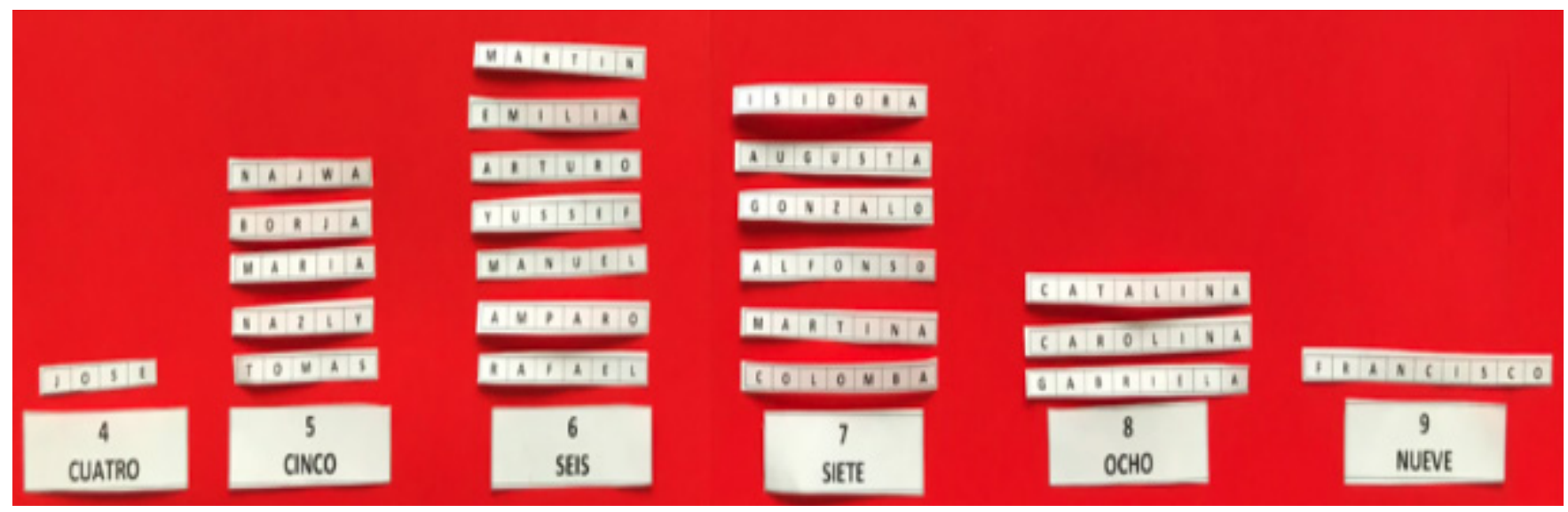

Experiencia 5: El día de la comida saludable

- Nivel: 4-5 años

- Contenidos implícitos: frecuencia absoluta, comparación de frecuencias.

- Materiales: láminas con situaciones cotidianas, tablero para el profesor y estudiantes.

- Habilidades y aprendizajes a lograr: En esta experiencia de aprendizaje se potencia, por un lado, la habilidad de orientarse temporalmente 
en situaciones cotidianas, empleando nociones y relaciones de frecuencia. Y por otro lado, emplear cuantificadores, tales como: “más que”, “menos que”, "igual que”, al comparar cantidades de objetos como representación de situaciones cotidianas.

- Gestión de la actividad:

A partir de diversas actividades referidas al "Día de la comida saludable", el profesor pregunta a los estudiantes ¿cuál es su fruta preferida? A partir de esta pregunta surgen diversas respuestas, lo que se aprovecha para hacer ver a los estudiantes el conjunto de todos los posibles resultados (manzana, naranja, frutilla y uva). Aunque no se introduce todavía el término de "espacio muestral", ya que es propio de niveles superiores, sí que es importante que los estudiantes empiecen a tener una primera idea intuitiva del conjunto de todos los posibles resultados de un experimento aleatorio. A continuación, el profesor pregunta a los estudiantes ¿cómo podemos saber cuál es la fruta preferida en el curso? Algunos estudiantes responden que contando cuántas hay de cada una. Ante esta respuesta, el profesor comienza a distribuir láminas con dibujos de las frutas preferidas mencionadas por los estudiantes (Figura 5).

Figura 5: Láminas de frutas entregadas a los estudiantes (archivo de los autores).
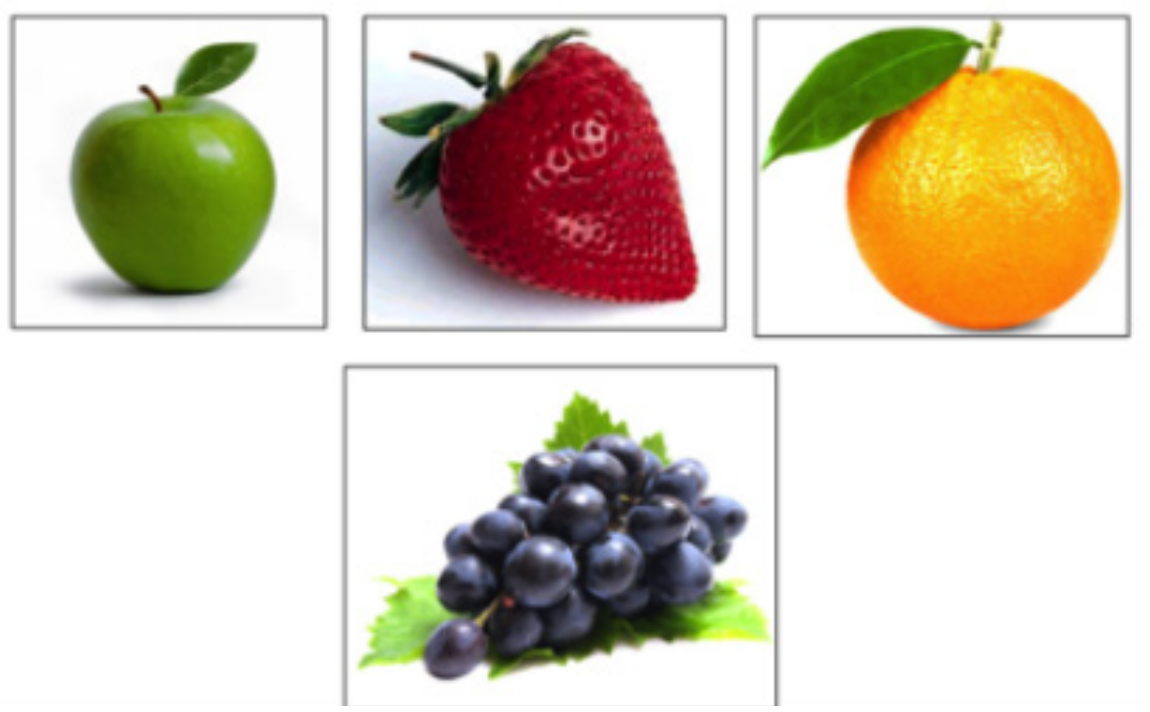

Una vez que cada estudiante tiene su lámina con su fruta preferida, el profesor solicita que organicen o representen las respuestas del curso. Los estudiantes forman grupos de acuerdo con sus preferencias, contabilizan cuántos prefieren cada tipo de fruta y se les plantea la pregunta ¿cuántos estudiantes prefieren cada fruta? Con el propósito de fomentar la representación, el profesor entrega un cubo multiencaje a cada niño para que registren de manera concreta y 
vertical la fruta que cada uno prefiere (Figura 6), y les indica que el cubo de color rojo es para aquellos estudiantes cuya fruta preferida es la frutilla, el verde para quienes prefieren la manzana, el azul para los que prefieren las uvas y el amarillo para los que prefieren las naranjas.

Figura 6: Registren de manera concreta cada fruta (archivo de los autores).

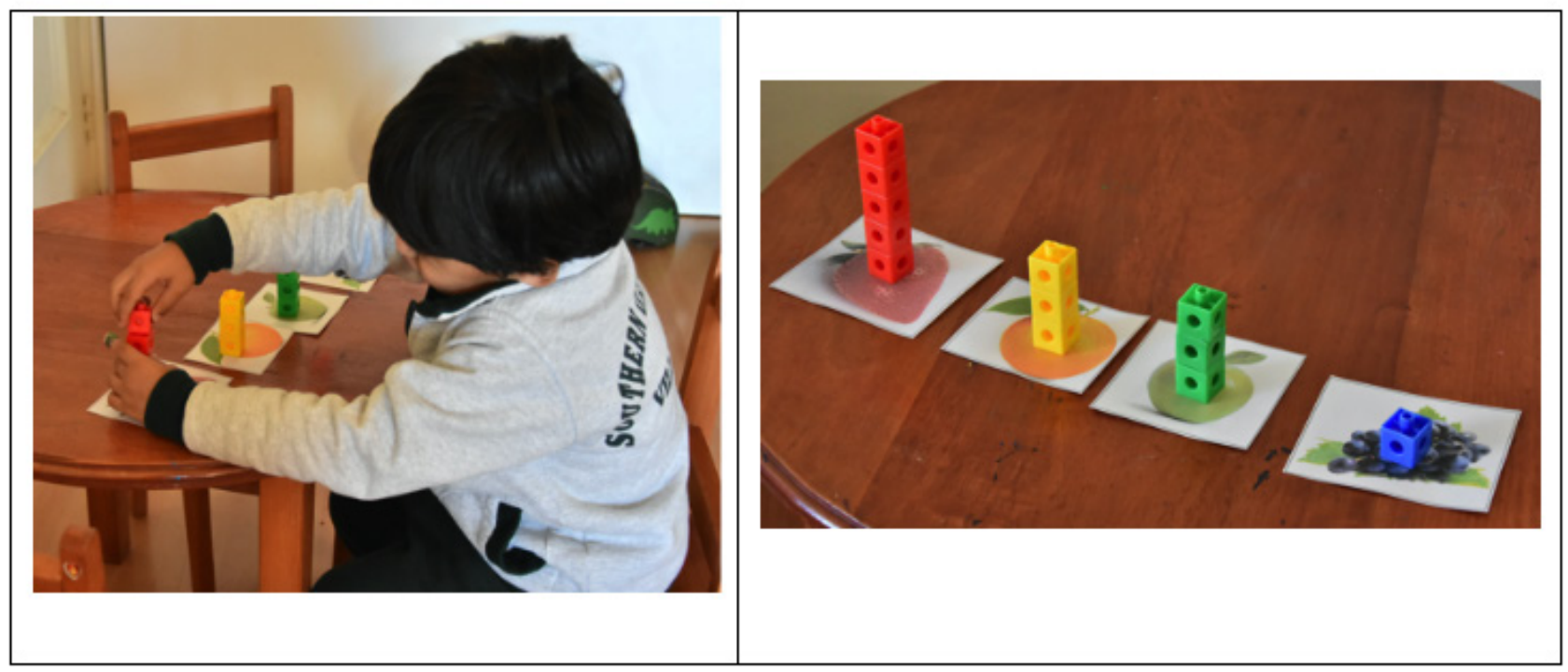

Como se observa en la Figura 6, siguiendo las orientaciones del profesor, los estudiantes apilan los bloques multiencaje según su fruta de preferencia. Como puede apreciarse en dicha figura, este tipo de gráfico proporciona elementos visuales para que los estudiantes puedan tener una primera idea intuitiva del concepto de frecuencia absoluta de cada valor de la variable (en este caso, cualitativa), lo que les permitirá más adelante dar paso a representaciones más abstractas.

\section{RefLeXiones finales}

En este artículo hemos presentado algunas orientaciones didácticas y recursos a utilizar en el aula de Educación Infantil para llevar a cabo una enseñanza eficaz de la estadística y la probabilidad en las primeras edades. Una primera reflexión importante es, pues, decidir cómo se plantea y gestiona la enseñanza de la estadística y la probabilidad en los primeros niveles escolares.

En la propuesta que se ha presentado y que sigue los planteamientos de organizaciones y autores de reconocido prestigio (e.g., GODINO; BATANERO; CAÑIZARES, 1987; NCTM, 2003; BATANERO; GODINO, 2004) se plantea que, en lugar de llevar a cabo una enseñanza centrada en la transmisión, la 
repetición y la práctica, la enseñanza debería plantearse a partir de situaciones contextualizadas que permitan un aprendizaje inductivo de los conceptos, impulsando las conexiones necesarias con la propia experiencia (ALSINA, 2012). Este planteamiento conlleva la necesidad de que para aprender conocimientos de estadística y probabilidad, es mucho más eficaz involucrar a los estudiantes en investigaciones estadísticas (recogida, organización, representación e interpretación de datos) que les conduzcan a la necesidad de conocer este tipo de datos para poder obtener conclusiones.

En definitiva, en las primeras edades (ni tampoco en los niveles superiores) no se trata de informar acerca del nombre del concepto, transmitir el conocimiento matemático formal asociado a dicho concepto y después practicarlo en situaciones descontextualizadas, sino de provocar la necesidad de que surjan ideas matemáticas intuitivas a partir de la propia experiencia, de manera que se construya el concepto de forma inductiva.

En consecuencia, es necesario que los profesores ofrezcan a sus estudiantes experiencias a partir de situaciones cotidianas que despierten su curiosidad innata, donde a través de las distintas etapas del ciclo de investigación (en el caso de la estadística) y de la cuantificación de la incertidumbre (en el caso de la probabilidad) éstos sean capaces de construir de manera paulatina una adecuada comprensión de la estadística y la probabilidad desde las primeras edades. Este es el gran cambio metodológico que, sin duda, contribuirá a desarrollar una mayor alfabetización estadística y probabilística de nuestros estudiantes.

No se trata de conocer a fondo las teorías respectivas, cosa reservada a especialistas, sino de educar la intuición para que no parezcan cosas caprichosas ni milagrosas. Tal vez muchos de los inconvenientes del comportamiento global de grandes sectores de la población provengan de que la gran mayoría de los ciudadanos no han sido nunca educados en probabilidad y estadística. (SANTALÓ,1977) 


\section{Statistical and Probabilistic Literacy: First Steps to Developing It from Early Childhood Education}

Abstract: This study explores the fundamental ideas linked to statistical and probabilistic literacy which can be developed since early childhood education. To that end, we analyze the explicit learning expectations found in the American and Chilean curricular guidelines for this level and compared them with the cognitive and disposition components described in the statistical and probabilistic literacy model. Likewise, we provide examples of different contexts for developing statistical and probabilistic literacy at youngest school ages, as a way of initiating children into critical thinking. Finally, the study offers some guidelines that can be used to support teacher education.

Keywords: Statistical education. Continuing education. Infant Education. 


\section{Alfabetização estatística e probabilística: primeiros passos para o desenvolvimento desde a educação infantil}

Resumo: Neste trabalho, indagamos sobre as ideias fundamentais ligadas à alfabetização estatística e probabilística que podem ser desenvolvidas desde a educação infantil. Para isso, analisamos as expectativas de aprendizado explícitas nas diretrizes curriculares americanas e chilenas para o referido nível educativo, as quais contrastam com os componentes cognitivos e de disposição presentes no modelo de alfabetização estatística e probabilística. Além disso, são exemplificadas diferentes situações para o desenvolvimento da alfabetização estatística e probabilística nas primeiras idades, como uma maneira de iniciar as crianças no pensamento crítico. Concluímos com algumas orientações que podem servir de apoio para a formação de professores.

Palavras-chave: Educação estatística. Formação de professores. Educação infantil. 


\section{AgRADECIMIENTOS}

Trabajo realizado en el marco del proyecto FONDECYT INICIACIÓN № 11150412 financiado por la Comisión Nacional de Investigación Científica y Tecnológica de Chile.

\section{REFERENCIAS BIBLIOGRÁFICAS}

AIZIKOVITSH-UDI, E.; KUNTZE, S. Critical thinking as an impact factor on statistical literacy. In: MAKAR, K.; DE SOUSA, B.; GOULD, R. (Eds.). Sustainability in statistics education. Proceedings of the Ninth International Conference on Teaching Statistics. Voorburg: International Statistical Institute, 2014, p. 1-6.

ALSINA, Á. La estadística y la probabilidad en educación infantil: conocimientos disciplinares, didácticos y experienciales. Didácticas Específicas, Madrid, n. 7, p. 4-22, dic. 2012.

ALSINA, Á. Diseño, gestión y evaluación de actividades matemáticas competenciales en el aula. Épsilon. Revista de Educación Matemática, Cádiz, v. 33, n. 92, p. 7-29, 2016.

ALSINA, Á. Contextos y propuestas para la enseñanza de la estadística y la probabilidad en Educación Infantil: un itinerario didáctico. Épsilon. Revista de Educación Matemática, Cádiz, v. 34, n. 95, p. 25-48, 2017.

ALSINA, Á.; VÁSQUEZ, C. De la competencia matemática a la alfabetización probabilística en el aula: elementos para su caracterización y desarrollo. UNIÓN. Revista Iberoamericana de Educación Matemática, São Paulo, n. 48, p. 41-58, 2016.

ARTEAgA, P.: BATANERO, C.; CONTRERAS, J. M. Gráficos estadísticos en la educación primaria y la formación de profesores. INDIVISA. Boletín de Estudios e Investigación, Madrid, n. 12, p. 123-136, 2011.

ARTEAGA, P.; BATANERO, C.; CAÑADAS, G.; CONTRERAS, J. M. Las tablas y gráficos estadísticos como objetos culturales. NÚMEROS. Revista de Didáctica de las Matemáticas, Tenerife, n. 76, p. 55-67, 2011.

BATANERO, C.; ARTEAGA, P.; RUIZ, B. Análisis de la complejidad semiótica de los gráficos producidos por futuros profesores de educación primaria en una tarea de comparación de dos variables estadísticas. Enseñanza de las Ciencias, Barcelona, v. 28. n. 1, p. 141-154, 2010. 
BATANERO, C.; GODINO, J. D. Estocástica: estadística y probabilidad. In: GODINO, J. D. (Ed.). Didáctica de las matemáticas para maestros. Granada: Universidad de Granada, 2004, p. 405-455.

FRANKLIN, C.; KADER, G.; MEWBORN, D.; MORENO, J.; PECK, R.; PERRY, M.; SCHEAFFER, R. Guidelines for assessment and instruction in statistics education (GAISE) report: A Pre-K- 12 curriculum framework. Alexandria: American Statistical Association, 2005.

GAISE. Guidelines for assessment and instruction in statistics education. College report. Alexandria: American Statistical Association, 2016.

GAL, I. Towards 'probability literacy' for all citizens. In: JONES, G. (Ed.). Exploring probability in school: Challenges for teaching and learning. Nueva York: Kluwer, 2005, p. 43-71.

GAL, I. Adults' Statistical literacy: meanings, components, responsibilities. International Statistical Review, Nederlandn, n. 70, p. 1-25, 2002.

GAL, I. Developing probability literacy: needs and pressures stemming from frameworks of adult competencies and mathematics curricula. In: $\mathrm{CHO}, \mathrm{S}$. J. (Ed.). Proceedings of the 12th International Congress on Mathematical Education. New York: Springer, 2012, p. 1-7.

GODINO, J.; BATANERO, C.; CAÑIZARES, M. J. Azar y probabilidad. Fundamentos teóricos y propuestas curriculares. Madrid: Editorial Síntesis, 1987.

MINEDUC. Bases curriculares para la Educación Parvularia. Santiago: Unidad de Currículum y Evaluación, 2018.

MINEDUC. Ley General de Educación. Santiago: Unidad de Currículum y Evaluación, 2009.

NCTM. Curriculum and evaluation standards for school mathematics. Reston, VA: The National Council of Teachers of Mathematics, 1989.

NCTM. Principles and standards for school mathematics. Reston, VA: The National Council of Teachers of Mathematics, 2000.

NCTM. Principios y estándares para la educación matemática. Sevilla: SAEM Thales, 2003.

NCTM. De los principios a la acción. Para garantizar el éxito matemático para 
todos. Reston, VA: The National Council of Teachers of Mathematics, 2015.

SANTALÓ, L. A. La educación matemática hoy. Barcelona: Teide, 1997.

VÁSQUEZ, C. Surgimiento del Lenguaje Probabilístico en el Aula de Educación Primaria. REnCiMa, v. 9, n. 2, p. 374-389, 2018.

VÁSQUEZ, C.; ALSINA, A. Lenguaje probabilístico: un camino para el desarrollo de la alfabetización probabilística. Un estudio de caso en el aula de Educación Primaria. BOLEMA. Boletim de Educação Matemática, v. 31, n. 57, p. 454-478, 2017.

WATSON, J. Curriculum expectations for teaching science and statistics. In: MAKAR, K.; DE SOUSA, B.; GOULD, R. (Eds.). Sustainability in statistics education. Proceedings of the Ninth International Conference on Teaching Statistics. Voorburg: International Statistical Institute, 2014, p. 1-6. 


\section{SOBRE LOS AUTORES}

Claudia Vásquez Ortiz es doctora en educación por la Universidad de Girona (UdG). Actualmente es académica del Departamento de Matemáticas del campus Villarrica de la Pontificia Universidad Católica de Chile (PUC).

E-mail : cavasque@uc.cl

Danilo Díaz-Levicoy es máster en didáctica de la matemática por la Universidad de Granada (UGR), España. Actualmente es doctorando en Ciencias de la Educación de la Universidad de Granada (UGR), Granada, España.

E-mail: dddiaz01@hotmail.com

Claudia Coronata es doctora en educación por la Universidad de Girona (UdG). Actualmente es académica del Departamento de Matemáticas del campus Villarrica de la Pontificia Universidad Católica de Chile (PUC).

E-mail: ccoronata@uc.cl

Ángel Alsina es doctor en psicología por la Universidad Autónoma de Barcelona (UAB). Actualmente es catedrático de didáctica de la matemática de la Universidad de Girona (UdG), Girona, España.

E-mail: angel.alsina@udg.edu

Recebido em: maio de 2018

Aprovado em: junho de 2018 